\title{
Accessory gene regulator (agr) dysfunction was unusual in Staphylococcus aureus isolated from Chinese children
}

Xin Yang ${ }^{1 \dagger}$, Fang Dong ${ }^{2 \dagger}$, Suyun Qian ${ }^{14^{*}} \mathbb{D}$, Lijuan Wang ${ }^{1}$, Yingchao Liu ${ }^{1}$, Kaihu Yao ${ }^{3}$, Wenqi Song ${ }^{2}$, Jinghui Zhen ${ }^{2}$, Wei Zhou', Hong $\mathrm{Xu}^{2}$ and Hongyan Zheng ${ }^{2}$

\begin{abstract}
Background: Staphylococcus aureus (S. aureus) with accessory gene regulator (agr) dysfunction occurs in health care settings. This study evaluated the prevalence and the molecular and drug resistance characteristics of $\mathrm{S}$. aureus with dysfunctional agr in a pediatric population in Beijing, China.

Results: A total of 269 nonduplicate S. aureus clinical isolates were isolated from Beijing Children's Hospital, including 211 methicillin-resistant S. aureus (MRSA) from September 2010-2017 and 58 methicillin-sensitive S. aureus (MSSA) from February 2016-2017. Only 8 MRSA and 2 MSSA isolates were identified as agr dysfunction, and the overall prevalence rate was 3.7\%. For MRSA isolates, ST59-SCCmec IV and ST239-SCCmec III were the most common clones, and the prevalence rate of agr dysfunction in ST239-SCCmec III isolates (17.39\%) was significantly higher than in ST59-SCCmec IV (1.69\%) and other genotype strains $(P=0.006)$. Among the agr dysfunctional isolates, only one MRSA ST59 isolate and one MSSA ST22 isolate harbored pvl. No significant difference was detected between agr dysfunction and agr functional isolates regarding the biofilm formation ability $(P=0.4972)$; however, 9/10 agr dysfunctional isolates could effectuate strong biofilm formation and multidrug resistance. Among MRSA, the nonsusceptibility rates to ciprofloxacin, gentamicin, and trimethoprim-sulfamethoxazole were significantly higher in agr dysfunctional isolates than in isolates with functional agr $(P<0.05)$. Two isolates belonging to ST239 had no mutations in agr locus, but a synonymous mutation was found in agrA in another ST239 isolate. The inactivating mutations were detected in other seven agr dysfunctional isolates. The variants were characterized by nonsynonymous changes $(n=5)$ and frameshift mutations (insertions, $n=2)$, which mainly occurred in agrC and agrA.

Conclusions: The results showed that agr dysfunctional S. aureus was not common in Chinese children, and ST59SCCmec IV was associated with lower prevalence of agr dysfunction as compared to ST239-SCCmec III isolates. The agr dysfunctional isolates were healthcare-associated, multidrug resistant and form strong biofilm, which suggested that agr dysfunction might offer potential advantages for $S$. aureus to survive in a medical environment.
\end{abstract}

Keywords: Staphylococcus aureus, Accessory gene regulator, Children, China

\footnotetext{
* Correspondence: syaian1211@163.com

${ }^{+}$Xin Yang and Fang Dong contributed equally to this work.

'Pediatric Intensive Care Unit, Beijing Children's Hospital, Capital Medical

University, National Center for Children's Health, No.56 Nan-Li-Shi Road,

Beijing 100045, China

${ }^{4}$ Present address: No. 56 Nan-li-shi Road, Beijing 100045, China

Full list of author information is available at the end of the article
}

(c) The Author(s). 2019 Open Access This article is distributed under the terms of the Creative Commons Attribution 4.0 International License (http://creativecommons.org/licenses/by/4.0/), which permits unrestricted use, distribution, and reproduction in any medium, provided you give appropriate credit to the original author(s) and the source, provide a link to the Creative Commons license, and indicate if changes were made. The Creative Commons Public Domain Dedication waiver (http://creativecommons.org/publicdomain/zero/1.0/) applies to the data made available in this article, unless otherwise stated. 


\section{Background}

Staphylococcus aureus (S. aureus) continues to be a leading cause of both community-and healthcare-associated infections, including skin and soft tissue infections, bacteremia, pneumonia, osteomyelitis, and endocarditis. Virulence factors play a major role in the pathogenesis of $S$. aureus, such as Panton-Valentine leukocidin (PVL). PVL is a pore-forming exotoxin composed of LukS-PV and LukF-PV. The PVL-producing strains have been associated with the onset of skin and soft tissue infections (SSTIs) and can also cause severe invasive infections (necrotizing pneumonia, etc) [1]. In addition, S. aureus can form biofilms not only in biological samples and surfaces of medical devices but also in tissues [2]. Biofilm can protect $S$. aureus from the damage of antibiotics and the host immune system [3]. Subsequently, the successful eradication of $S$. aureus infections is difficult, rendering biofilm as a vital factor in chronic infections.

The accessory gene regulator (agr)-mediated quorum sensing plays a major role in staphylococcal pathogenesis, which can downregulate the expression of cell surface-associated proteins (microbial surface components recognizing adhesive matrix molecules [MSCRAMMs]) and upregulate the expression of extracellular toxin (hemolysins, enterotoxins, extracellular proteases, etc.) [4]. The regulation of virulence factors by $a g r$ is important for disease progression. Several studies demonstrated that genetically engineered agr-knockout strains had attenuated virulence in animal models of skin and soft tissue infections, pneumonia, infective endocarditis, arthritis, and osteomyelitis [5-10].

However, recent evidence indicated that agr dysfunction exists extensively in healthcare settings (13-82\%) [11-14], which might be attributed to the agr dysfunction that confers a potential advantage for $S$. aureus in the current medical environment [15]. For example, agr dysfunction has been linked to attenuated vancomycin activity; both laboratory-derived vancomycin-intermediate $S$. aureus (VISA) and clinical VISA isolates developed during vancomycin therapy often exhibit as agr dysfunction [16]. In addition, infections, such as bacteremia and bone and joint infection, caused by strains with dysfunctional $a g r$ often manifest as a chronic course and result in adverse outcomes [17-19]. Thus, understanding the prevalence of agr dysfunction in a specific area is essential.

Herein, we conducted a molecular epidemiology study in Beijing Children's Hospital in China. The primary objectives of the present study were as follows: (1) to detect the prevalence of agr dysfunction in MRSA and MSSA isolates; (2) to explore the molecular characteristics, $p v l$ carriage rate, biofilm formation ability, and antibiotic susceptibility of $S$. aureus isolated from pediatric patients; these indicators were compared mainly based on agr functionality.

\section{Methods}

\section{Bacterial isolates}

This study was approved by the Ethics Committee of Beijing Children's Hospital affiliated to the Capital Medical University (No. 2016-93, 23/06/2016), and obtained clearance from the Institutional Biosafety Committee (IBSC) ([2017] No.43). S. aureus strains were collected and identified as follows. If the clinical samples (blood, pleural effusion, and joint effusion, etc.) were obtained from steril specimens (blood, pleural effusion, bone marrow, cerebrospinal fluid, joint effusion, seroperitoneum, etc), the bacterial growth in the culture medium could be directly identified by VITEK $^{\oplus}$ MS system (BioMérieux, France). If the clinical samples were obtained from the non-sterile specimens (respiratory tract, skin, etc.), several suspected colonies were selected according to the morphological characteristics and identified by VITEK $^{\oplus}$ MS system. The coagulase test and detection of nuc gene were employed to identify $S$. aureus as described previously by Kateete et al. [20] and Petersson et al. [21], respectively; these isolates were further confirmed to be $S$. aureus. The MRSA isolates were screened by cefoxitin disc (30 mg, Oxoid) diffusion test, while the polymerase chain reaction (PCR) was employed for the detection of the $m e c A$ gene [22]. In the case of strains isolated from the same patient, if the genotyping studies revealed identical genotype, only one of them was included in the study, which ensured that all strains involved in the current study were non-repetitive. All the strains were preserved at $-80{ }^{\circ} \mathrm{C}$ in a bacterial cryopreservation reagent comprised of $2.5 \%$ TSB (w/v), $16.7 \%$ glycerol (v/v), and $66.7 \%$ sterile horse serum (v/v).

The clinical data of children, including age, sex, medical history, isolation site, infection sites, and medication use, were collected. S. aureus infections were categorized as healthcare-associated (HA) or community-associated (CA) according to the epidemiology definitions established previously [23].

\section{Delta-hemolysin expression}

RNAIII is the major effector molecule of agr system, and also encodes the gene for delta-hemolysin ( $h l d$ ) [24]. Thus, $\delta$-hemolysin production was used to assess the function of agr operon. The expression of $\delta$-hemolysin was determined using $S$. aureus RN4420, which produced only $\beta$-hemolysin without the interference of $\alpha$ or $\delta$ - hemolysins. Furthermore, $\beta$-hemolysin and $\delta$-hemolysin have synergetic effects, while $\beta$-hemolysin inhibits lysis by $\alpha$-hemolysin [25]. Therefore, the presence of enhanced hemolysis within the $\beta$-hemolysin zone of RN4220 indicates the production of $\delta$-hemolysin by the test strains. 


\section{agr sequencing}

The agr locus of non-hemolytic isolates was amplified using the method reported by Robinson et al. [26]. In addition, agr-w1f (5'-CCATTTgCCCAATTCATTC-3') was used for sequencing the PCR product amplified by agrX1F ( $5^{\prime}$-TCGT ATAATGACAGTGAGGAGAGT-3') and $\operatorname{agrCD} 2434 \mathrm{dn}$ (5'-TAATACCAATACTGCGACTT-3'). The resultant sequences were compared with the known agr sequences of $S$. aureus strain from the appropriate agr specificity group and sequence types (STs), including NCTC8325 (CC8) (CP000253.1), SA957 (ST59) (NC_022442.1), ST398 (NC_017333.1), N315 (agr group II), and H-EMRSA-15 genome (ST22) (CP007659.1).

\section{Molecular genotyping analysis}

Multilocus sequence typing (MLST) was performed as described by Enright et al. [27]. The allelic profiles (allele numbers) and ST types were determined based on the MLST database (http://saureus.mlst.net/). The staphylococcal protein A (spa) gene repeat region was amplified and sequenced as described previously [28], and the sequencing data were submitted to the $S$. aureus spa type database (http://spaserver.ridom.de) to determine the spa type. Agr typing was assigned by multiplex PCR according to the method described by Gilot et al. [29]. COL (agr I), N315 (agr II), TY114 (agr III), and A920210 were used as positive controls.

The staphylococcal cassette chromosome mec (SCCmec) types of MRSA isolates were determined using a multiplex PCR as described previously [30]. The reference strains used for SCCmec typing included NCTC10442 (SCCmec I), N315 (SCCmec II), 85/2082 (SCCmec III), JCSC4744 (SCCmec IV), and IMVS 67 (SCCmec V).

\section{Detection of $p v l$ gene}

The detection of $p v l$ was carried out using primers and conditions as described by Jarraud et al. [31]. ATCC25923 was used as a positive control.

\section{Biofilm formation assays}

Tissue culture plate method (TCP) was used to assess the biofilm forming ability of the nonhemolytic strains, as described in our previous study [32]. Briefly, overnight cultures in tryptic soy broth (TSB) (OXOID, USA) containing $0.25 \%$ glucose were adjusted to $10^{6} \mathrm{CFU} / \mathrm{mL}$. Then, $0.2 \mathrm{~mL}$ cell suspension was inoculated into each well of 96-well flat-bottom plates (Corning Costar \#3599, USA) at $37^{\circ} \mathrm{C}$ for $48 \mathrm{~h}$. Subsequently, the wells were washed two times with normal saline, fixed by methanol for $15 \mathrm{~min}$, stained with $0.1 \%$ crystal violet for $5 \mathrm{~min}$, rinsed, and air-dried. The stained biofilm was solubilized with $33 \%$ glacial acetic acid, and the optical density (OD) was measured at $590 \mathrm{~nm}$ using on a
CLARIOstar Microplate reader (BMG LABTECH, Germany). Each isolate was tested in triplicates. The negative control wells contained only the broth. The cut-off $\mathrm{OD}$ value (ODc) was defined as an average OD of negative control with three times of standard deviation. The biofilm formation ability was classified as negative $(\mathrm{OD} \leq \mathrm{ODc})$, weak (ODc $<\mathrm{OD} \leq 2 \mathrm{ODc}, \mathrm{WBF})$, moderate (2ODc $<\mathrm{OD} \leq \mathrm{ODc}, \mathrm{MBF})$, and strong (4ODc $<\mathrm{OD}, \mathrm{SBF})$.

\section{Antimicrobial susceptibility testing}

Antimicrobial susceptibility testing to 12 antimicrobial agents (penicillin G, oxacillin, erythromycin, clindamycin, tetracycline, gentamicin, chloramphenicol, ciprofloxacin, rifampin, linezolid, vancomycin, mopiroxacin; National Institutes for Food and Drug Control, China) were performed by agar dilution method as described by Wiegand et al. [33] with slight modification. Mueller-Hinton Agar (MHA; OXOID CM0337B, UK) medium without cation-adjustments were prepared and autoclaved according to the manufacture's instructions. The antibacterial drugs were diluted for usage according to the Clinical and Laboratory Standards Institute (CLSI) guidelines [34]. Appropriate MHA medium was cooled to about $50{ }^{\circ} \mathrm{C}$ and poured to the $15 \times 100 \mathrm{~mm}$ petri dish to produce the required depth of $3-4 \mathrm{~mm}$. The final antibiotic concentration for each drug ranged from $0.032-256 \mathrm{mg} / \mathrm{L}$ and three control agar plates were without any antibiotic. The inoculation was carried out using a multipoint inoculator. Bacterial suspension, at a density of $1 \times 10^{7} \mathrm{CFU} / \mathrm{mL}$, was inoculated to the agar plates starting from the lowest concentration. Then, the inoculum spots were dried at room temperature before inverting the plates and incubated at $35{ }^{\circ} \mathrm{C}$ for $16-20 \mathrm{~h}$ before obtaining the minimal inhibitory concentration (MIC). In addition, the E-test method was used to determine the MIC of all isolates to sulphamethoxazole/trimethoprim (SXT) (BioMeriuex, France). MHA plates were inoculated by streaking the standardized inoculums (0.5 McFarland, about $1.5 \times 10^{8} \mathrm{CFU} / \mathrm{mL}$ with a sterile swab. The SXT E-test strips (BioMeriuex) were placed on the plates, followed by incubation at $35^{\circ} \mathrm{C}$ for $16-20 \mathrm{~h}$.

The MIC reading for both E-test and agar dilution method was conducted independently by a senior experimenter, with the result confirmed by a second reader. The results of MIC were interpreted according to the CLSI breakpoints for Staphylococcus spp. [34]. S. aureus ATCC29213 was used as quality control. Multidrug resistance (MDR) was defined as isolates resistant to $\geq 3$ classes of non- $\beta$-lactam antimicrobials for MRSA, and resistant to $\geq 3$ classes of antibiotics including $\beta$-lactam antibiotics for MSSA. 


\section{Statistical analysis}

SAS JMP Statistical Discovery v11.0 was used for statistical analysis. Chi-squared $\left(\chi^{2}\right)$ test or Fisher's exact test was used to analyze the categorical variables, and Wilcoxon rank sum test was used to compare the biofilm formation ability between the two groups. $P<0.05$ was considered as statistically significant.

\section{Results}

\section{Clinical characteristics}

A total of 269 non-duplicate $S$. aureus clinical isolates were collected from Beijing Children's Hospital, including 211 MRSA from September 2010-2017 and 58 MSSA from February 2016-2017. These strains were isolated from several clinical sources, including respiratory tract (6 from throat swab, 69 from sputum, and 37 from bronchial alveolar lavage fluid), skin and soft tissue (38 from pus, 20 from secretions of omphalitis, 10 from skin secretions, 17 from wound surface, 6 from eye secretions, and 2 from ear secretions), sterile sites (48 from blood, 6 from pleural effusion, 3 from bone marrow, 3 from cerebrospinal fluid, 2 from joint effusion, and 1 from seroperitoneum), and midstream urine (1 isolate). The characteristics of patients (107 females and 162 males; median age: 11.9 months) from whom samples were collected are shown in Table 1. Approximately $72.86 \%(196 / 269)$ of the patients were $<3$-years-old. The proportion of children with community-associated (CA) infections and hospital-associated infections was roughly equivalent (50.19\% vs. $49.81 \%)$. Then, 9/10 agr dysfunctional isolates caused healthcare-associated (HA) infections. A total of $29.74 \%(80 / 269)$ children presented invasive infections. All children included in this study were treated with antibiotics. Vancomycin and linezolid were used in $24.54 \%(66 / 269)$ and $16.73 \%$ (45/269) patients, respectively. The median hospital stay was 15 (interquartile range, IQR: 10-24) Days.

\section{Molecular typing and virulence characteristics}

Among the 269 isolates, only 3.79\% (8/211) MRSA and $3.45 \%$ (2/58) MSSA isolates were identified with agr dysfunction (no apparent hemolytic activity as shown in Fig. 1), and the overall prevalence rate was $3.71 \%$ (10/269).

The molecular characteristics of MRSA and MSSA stratified by agr functionality were shown in Table 2 and Table 3, respectively. ST59-SCCmec IV-t437/t441-agr I $(49.29 \%, 104 / 211)$ and ST239-SCCmec III-t030/t037-agr I $(9.00 \%, 19 / 211)$ were the most common MRSA clones. The prevalence rate of $a g r$ dysfunction in ST239-SCCmec III isolates $(17.39 \%, 4 / 23)$ was significantly higher than in ST59-SCCmec IV $(1.69 \%, 2 / 118)$ and other genotype strains $(2.86 \%, 2 / 70)(P=0.006)$ (Table 1). For MSSA isolates, the top 4 genotypes were ST22-t309-agr I (15.52\%, 9/58), ST398-t571-agr I

Table 1 Characteristics of patients for samples

\begin{tabular}{|c|c|c|c|c|}
\hline Patient characteristics & Total & Dysfunctional agr $(N=10)$ & Functional agr $(N=159)$ & $P$-value \\
\hline Male sex, N (\%) & $162(60.22)$ & $5(50.00)$ & $157(60.62)$ & 0.5253 \\
\hline Age (months), median $\left(\mathrm{IQR}^{a}\right)$ & $11.9(1.27-55.14)$ & $33.27(13.15-108.38)$ & $11.33(1.17-54.90)$ & 0.0797 \\
\hline Age distribution & & & & 0.1581 \\
\hline$\leq 28$ days & $59(21.93)$ & 0 & $59(22.78)$ & \\
\hline 29 days -3 years & $137(50.93)$ & $7(70.00)$ & $130(50.19)$ & \\
\hline $4-6$ years & $28(10.41)$ & 0 & $28(10.81)$ & \\
\hline $7-15$ years & $45(16.73)$ & $3(30.00)$ & $42(16.22)$ & \\
\hline Origin, N (\%) & & & & 0.0102 \\
\hline CA & $135(50.19)$ & $1(10.00)$ & $134(51.74)$ & \\
\hline HA & $134(49.81)$ & $9(90.00)$ & $125(48.26)$ & \\
\hline Disease & & & & 0.2900 \\
\hline Invasive infection ${ }^{b}, \mathrm{~N}(\%)$ & $80(29.74)$ & $1(10.00)$ & $9(90.00)$ & \\
\hline Non-invasive infection ${ }^{c}, \mathrm{~N}(\%)$ & $189(70.26)$ & $79(30.50)$ & $180(69.50)$ & \\
\hline Vancomycin treatment, N (\%) & $66(24.54)$ & $2(20.00)$ & $64(24.71)$ & 1.0000 \\
\hline Linezolid treatment, N (\%) & $45(16.73)$ & $2(20.00)$ & $43(16.60)$ & 0.6758 \\
\hline Hospitalization-Median (IQR) & $15(10-24)$ & $21(10.25-30.25)$ & $14(10-23)$ & 0.4000 \\
\hline
\end{tabular}

${ }^{a} Q R$ interquartile range

${ }^{b} S S T I$ Including skin and soft tissue infection, BSI bloodstream infection, CNSI central nervous system infection, IE infective endocarditis, $B J /$ bone and joint infection, SP severe pneumonia, Al intra-abdominal infection. Details were as follows: SSTI (1 case), AI (1 case), CNSI (1 case), BJI (3 cases), SP (11 cases), BSI (11 cases), BSI + SSTI (16 cases), BSI + AI (1 case), BSI + IE (4), BSI + BJI (6 cases), BSI + CNSI (2 cases), BSI + SP (5 cases), BJ + SSTI (1 case), SP + SSTI (1 case), SP + BJ (1 case), BSI + SSTI+CNSI (1 case), BSI + CNSI+IE (1 case), BSI + SP + CNSI (1 case), BSI + SP + SSTI (3 cases), BSI + BJI + SSTI (7 cases), BSI + BJI + SSTI+CNSI (1 cases), BSI $+\mathrm{SP}+\mathrm{SSTI}+\mathrm{CNSI}(1$ case $)$

'Including SSTI (83 cases), pneumonia (103 cases), pneumonia+SSTI (2 cases), urinary system infection (1 case) 

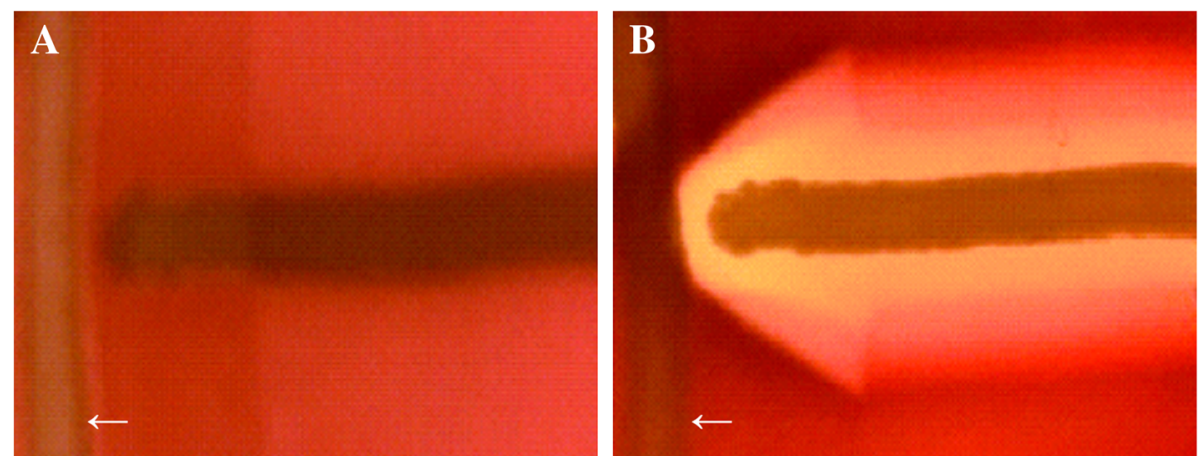

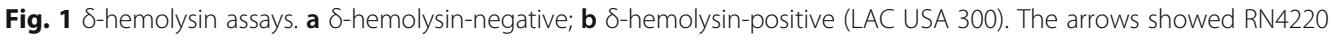

(10.34\%, 6/58), ST5-t002-agr II $(6.90 \%, \quad 4 / 58)$, ST6-t701-agr I $(6.90 \%, 4 / 58)$, and ST188-t189-agr I (6.90\%, 4/58).

The detection rate of $p v l$ was similar in MRSA $(35.55 \%, 75 / 211)$ and MSSA $(29.31 \%, 17 / 58)$ isolates $(P$ $=0.4359)$. Among MRSA, the $p v l$ prevalence rate of ST239-SCCmec III isolates $(4.35 \%, 1 / 23)$ was significantly lower than that of ST59-SCCmec IV (37.3\%, 44/ $118)$ and other strains $(42.86 \%, 30 / 70)(P=0.0031)$. All the ST22 isolates were $p v l$-positive. Among the ten agr dysfunctional isolates, only one MRSA belonging to the ST59-SCCmec IV-t437-agr I and one MSSA belonging to ST22-t310-agr I harbored $p v l$.

\section{Biofilm formation}

Table 4 shows that $88.63 \%(187 / 211)$ of MRSA and $56.90 \%(33 / 58)$ of MSSA isolates were strong biofilm formers. However, the biofilm formation ability of MRSA was significantly higher than that of MSSA isolates $(P<0.0001)$ (Fig. 2a). Interestingly, no significant difference was detected between agr dysfunction and $a g r$ functional isolates regarding the biofilm formation ability $(P=0.4972)$ (Fig. 2b); nevertheless, all the eight agr dysfunctional MRSA isolates and one MSSA isolate showed strong biofilm formation. Any association between $p v l$ and biofilm formation in both MRSA and MSSA isolates was not observed $(P=0.4004$ and $P=0.0509$, respectively) (Figs. 2c and d).

\section{Antimicrobial resistance}

The results of antimicrobial susceptibility test are shown in Table 5. In this study, the S. aureus isolates were sensitive to vancomycin and linezolid, but highly resistant to penicillin (97.03\%, 261/269) and erythromycin (98.89\%, 266/269). Approximately, 80.09\% (169/211) of MRSA and $74.14 \%(43 / 58)$ of MSSA isolates were MDR strains. The non-susceptibility rate of MRSA to oxacillin, clindamycin, tetracycline, and rifampin was significantly higher than that of MSSA isolates $(P<0.05)$. In addition, the non-susceptibility rates to ciprofloxacin, gentamicin, and SXT among MRSA isolates were significantly higher in agr dysfunctional isolates than in those with functional $a g r$.

\section{agr sequencing}

Two isolates that belonged to ST239 had no mutations in agr locus, and another ST239 isolate harbored a synonymous mutation in agrA. Inactivating mutations were detected in other 5 MRSA and 2 MSSA isolates. The variants were characterized by nonsynonymous changes $(n=5)$ and frameshift mutations (insertions, $n=2)$, which mainly occurred in $\operatorname{agrC}$ and $\operatorname{agr} A$ (Table 6).

\section{Discussion}

Recent evidence indicated that agr dysfunction was common among $S$. aureus clinical isolates, especially MRSA [11-14, 17, 18]. However, the dysfunction was unusual in pediatric populations in China according to the current study. Moreover, the data showed that infections caused by agr-dysfunctional strains were always HA. Similarly, the study by Shopsin et al. [35] showed that the carriage of an agr-defective strain was associated with hospitalization. Butterfield et al. [13] also indicated that agr dysfunction was closely associated with prior administration of $\beta$-lactam and fluoroquinolone. Therefore, the high antibiotic selection pressure in the medical environment might lead to the emergence of agr dysfunction, which might be due to the agr-controlled virulence that is energy-consuming and needs to be balanced with the expression of antibiotic resistance in a healthcare environment filled with antibiotics [36].

In the current study, MRSA strains showed strong homology, and ST59-SCCmec IV and ST239-SCCmec III were the most prevalent clones. This result was consistent with that of a previous study conducted by Qiao et al. in Chinese children [37]. These results showed that agr dysfunction was more common in ST239-SCCmec III than in ST59-SCCmec IV isolates, the phenomenon was consistent with a previous study, which indicated that SCCmec IV/IVa MRSA (3\%, known as CA-MRSA 
Table 2 Genotypic characteristics of MRSA isolates stratified by agr functionality

\begin{tabular}{|c|c|c|c|c|}
\hline \multirow[t]{2}{*}{ Genotype } & \multirow{2}{*}{$\begin{array}{l}\text { No. of } \\
\text { solates (\%) }\end{array}$} & \multicolumn{2}{|l|}{ agr } & \multirow{2}{*}{$\begin{array}{l}p v l(+), N \\
(\%)\end{array}$} \\
\hline & & Dysfunction & Function & \\
\hline Total & 211 & $8(3.79)$ & $203(96.21)$ & $75(35.55)$ \\
\hline \multicolumn{5}{|l|}{ Origins } \\
\hline Community-associated & $104(49.29)$ & 0 & $104(100.00)$ & $44(42.30)$ \\
\hline Hospital-associated & $107(50.71)$ & $8(7.47)$ & $99(92.52)$ & $31(28.97)$ \\
\hline \multicolumn{5}{|l|}{ MLST } \\
\hline 1 & $8(3.79)$ & 0 & $8(100.00)$ & $2(25.00)$ \\
\hline 22 & $7(3.32)$ & 0 & $7(100.00)$ & $7(100.00)$ \\
\hline 59 & $128(60.66)$ & $2(1.56)$ & $126(98.44)$ & $49(38.28)$ \\
\hline 88 & $8(3.79)$ & 0 & $8(100.00)$ & $5(62.50)$ \\
\hline 239 & $27(12.80)$ & $4(14.81)$ & $23(85.19)$ & $2(7.41)$ \\
\hline Others $^{a}$ & $33(15.64)$ & $2(6.06)$ & $31(93.94)$ & $10(30.30)$ \\
\hline \multicolumn{5}{|l|}{$\mathrm{sccmec}$} \\
\hline I & $1(0.47)$ & 0 & $1(100.00)$ & 0 \\
\hline III & $26(12.32)$ & $4(15.38)$ & $22(84.62)$ & $2(7.69)$ \\
\hline IV & $145(68.72)$ & $3(2.07)$ & $142(97.93)$ & $51(35.17)$ \\
\hline v & $32(15.17)$ & $1(3.13)$ & $31(96.87)$ & $19(59.38)$ \\
\hline$N T^{b}$ & $7(3.32)$ & 0 & $7(100.00)$ & $3(42.86)$ \\
\hline \multicolumn{5}{|l|}{ spa type } \\
\hline t030 & $16(7.58)$ & 0 & $16(100.00)$ & 0 \\
\hline t037 & $7(3.32)$ & $3(42.86)$ & $4(57.14)$ & $1(14.29)$ \\
\hline t309 & $7(3.32)$ & 0 & $7(100.00)$ & $7(100.00)$ \\
\hline t437 & $107(50.71)$ & $2(1.87)$ & $105(98.13)$ & $47(43.92)$ \\
\hline $\mathrm{t} 441$ & $14(6.64)$ & 0 & $14(100.00)$ & $7(50.00)$ \\
\hline Others $^{c}$ & $60(28.43)$ & $3(5.00)$ & 57 (095.00) & $13(21.67)$ \\
\hline \multicolumn{5}{|l|}{ agr type } \\
\hline । & $185(87.68)$ & $7(3.78)$ & $178(96.22)$ & $67(36.22)$ \\
\hline$\|$ & $6(2.84)$ & $1(16.67)$ & $5(83.33)$ & $1(16.67)$ \\
\hline III & $17(8.06)$ & 0 & $17(100.00)$ & $7(41.18)$ \\
\hline IV & $3(1.42)$ & 0 & $3(100.00)$ & 0 \\
\hline \multicolumn{5}{|l|}{ MLST-SCCmec type } \\
\hline ST1-SCCmec IV & $6(2.84)$ & 0 & $6(100.00)$ & 0 \\
\hline ST22-SCCmec V & $7(3.32)$ & 0 & $7(100.00)$ & $7(100.00)$ \\
\hline ST59-SCCmec IV & $118(55.92)$ & $2(1.69)$ & $116(98.31)$ & $44(37.29)$ \\
\hline ST239-SCCmec III & $23(10.90)$ & $4(17.39)$ & $19(82.61)$ & $1(4.35)$ \\
\hline Others & $57(27.01)$ & $2(3.51)$ & $50(96.49)$ & $23(40.35)$ \\
\hline \multicolumn{5}{|l|}{ MLST-SCCmec-spa-agr type } \\
\hline ST59-ScCmec IV-t437-agr I & $92(43.60)$ & $1(1.09)$ & $91(98.91)$ & $36(39.13)$ \\
\hline ST59-SCCmec IV-t441-agr I & $12(5.69)$ & 0 & $12(100.00)$ & $6(50.00)$ \\
\hline ST239-SCCmec III-t030-agr I & $12(5.69)$ & 0 & $12(100.00)$ & 0 \\
\hline ST239-SCCmec III-t037-agr I & $7(3.32)$ & $3(42.86)$ & $4(57.14)$ & 0 \\
\hline ST22-SCCmec V-t039-agr I & $7(3.32)$ & 0 & $7(100.00)$ & $7(100.00)$ \\
\hline Others & $81(38.39)$ & $4(4.94)$ & $77(95.06)$ & $26(32.10)$ \\
\hline
\end{tabular}

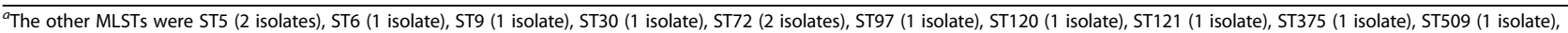
ST585 ( 1 isolate), ST630 ( 1 isolate), ST896 ( 1 isolate), ST950 (1 isolate), ST965 (2 isolates), ST1224 (1 isolate), ST1295 (1 isolate), ST1296 (1 isolate), ST1777 (1 isolate), ST1821 (1 isolate) ${ }^{b}$ Not determined

'The other spa types were t008 ( 1 isolate), t011 (1 isolate), t021 ( 1 isolate), t034 ( 2 isolates), t062 ( 2 isolates), t078 ( 2 isolates), t114 (6 isolates), t127 ( 3 isolates), t138 ( 1 isolate), t163 ( 1 isolate), t172 (4 isolate), t186 (1 isolate), t267 ( 1 isolate), t318 ( 1 isolate), t459 ( 2 isolate), t664 (1 isolate), t895 ( 2 isolates), t1894 (1 isolate), t1977 (1 isolate), t2270 (1 isolate), t2310 (1 isolate), t2755 (2 isolate), t3401 ( 1 isolate), t3515 ( 2 isolates), t3523 (1 isolate), t3590 (1 isolate), t4431 (1 isolate), t4549 (2 isolates), t7617 (1 isolate), t7637 (1 isolate), t8660 ( 2 isolates), t 8723 ( 1 isolate), t10555 (1 isolate), t12946 (1 isolate), t16365 (1 isolate)

clone) was associated with lower rates of agr dysfunction as compared to SCCmec I-III MRSA (43\%, known as HA-MRSA clone) [38]. Thus, the low prevalence of agr dysfunction in this study might be attributed to more than half of the MRSA isolates belonging to ST59 harboring the SCCmec IV.

In addition, infections caused by PVL-producing ST22-t309-agr I clone should be under intensive research. 
Table 3 Genotypic characteristics of MSSA isolates stratified by agr functionality

\begin{tabular}{|c|c|c|c|c|}
\hline \multirow[t]{2}{*}{ Genotype } & \multirow{2}{*}{$\begin{array}{l}\text { No. of } \\
\text { solates } \\
\text { (\%) }\end{array}$} & \multicolumn{2}{|l|}{ No. (\%) of isolates } & \multirow{2}{*}{$\begin{array}{l}\text { pvl } \\
\text { positive } \\
\text { [No. (\%)] }\end{array}$} \\
\hline & & Dysfunctional agr & Functional agr & \\
\hline Total & 58 & $2(3.45)$ & $56(96.55)$ & $17(29.31)$ \\
\hline Community-associated & $31(53.45)$ & $1(3.23)$ & $30(96.77)$ & $12(38.71)$ \\
\hline Hospital-associated & $27(46.55)$ & $1(3.70)$ & $26(96.30)$ & $5(18.52)$ \\
\hline \multicolumn{5}{|l|}{ MLST } \\
\hline 5 & $5(8.62)$ & 0 & $5(100.00)$ & 0 \\
\hline 7 & $5(8.62)$ & 0 & $5(100.00)$ & $1(20.00)$ \\
\hline 22 & $12(20.69)$ & $1(8.33)$ & $11(91.67)$ & $12(100.00)$ \\
\hline 25 & $6(10.34)$ & 0 & $6(100.00)$ & $1(16.67)$ \\
\hline 398 & $10(17.24)$ & $1(10.00)$ & $9(90.00)$ & $1(10.00)$ \\
\hline Others $^{a}$ & $20(34.48)$ & 0 & $20(100.00)$ & $2(10.00)$ \\
\hline \multicolumn{5}{|l|}{ spa type } \\
\hline t002 & $4(6.89)$ & 0 & $4(100.00)$ & 0 \\
\hline t189 & $4(6.89)$ & 0 & $4(100.00)$ & 0 \\
\hline t309 & $11(18.97)$ & 0 & $11(100.00)$ & $11(100.0)$ \\
\hline t571 & $6(10.34)$ & $1(16.67)$ & $5(83.33)$ & 0 \\
\hline t701 & $4(6.90)$ & 0 & $4(100.00)$ & 0 \\
\hline Others $^{b}$ & $29(50.00)$ & $1(3.45)$ & $28(96.55)$ & $6(20.69)$ \\
\hline \multicolumn{5}{|l|}{ agr type } \\
\hline । & $46(79.31)$ & $2(4.35)$ & $44(95.65)$ & $16(34.78)$ \\
\hline$\|$ & $9(15.52)$ & 0 & $9(100.00)$ & 0 \\
\hline III & $1(1.72)$ & 0 & $1(100.00)$ & 0 \\
\hline IV & $1(1.72)$ & 0 & $1(100.00)$ & 0 \\
\hline$N T^{c}$ & $1(1.72)$ & 0 & $1(100.00)$ & $1(100.00)$ \\
\hline \multicolumn{5}{|l|}{ MLST-spa-agr type } \\
\hline ST5-t002-agr II & $4(6.90)$ & 0 & $4(100.00)$ & 0 \\
\hline ST6-t701-agr I & $4(6.90)$ & 0 & $4(100.00)$ & 0 \\
\hline ST22-t309-agr I & $9(15.52)$ & 0 & $9(100.00)$ & $9(100.00)$ \\
\hline ST188-t189-agr I & $4(6.90)$ & 0 & $4(100.00)$ & 0 \\
\hline ST398-t571-agr I & $6(10.34)$ & $1(16.67)$ & $5(83.33)$ & 0 \\
\hline Others & $31(53.45)$ & $1(3.23)$ & $30(96.77)$ & $8(25.81)$ \\
\hline
\end{tabular}

${ }^{a}$ The other MLSTs were ST1 (1 isolate), ST6 (4 isolate), ST8 (1 isolate), ST15 (3 isolates), ST25 (6 isolates), ST59 (4 isolates), ST121 (1 isolate), ST188 (4 isolates), ST950 (1 isolate), ST1281 (1 isolate)

${ }^{b}$ The other spa types were t034 (3 isolates), t078 ( 2 isolates), t081 ( 1 isolate), t084 (3 isolates), t091 ( 2 isolates), t127 (1 isolate), t163 ( 1 isolate), t164 ( 1 isolate), t167 (1 isolate), t310 (1 isolate), t437 ( 2 isolates), t660 (1 isolate), t796 (2 isolates), t1062 (1 isolate), t1818 (1 isolate), t2092 (1 isolate), t4377 (1 isolate)

${ }^{\mathrm{C}}$ Not determined

The current literature demonstrated that ST22-MRSA isolates mainly carry SCCmec IV [39, 40]. However, in this study, ST22-t309-agr I-MRSA strains were classified as SCCmec V. Moreover, ST22-t309-agr I present in MSSA isolates indicated that ST22-t309-MRSA probably arose from ST22-t309-MSSA.

Furthermore, S. aureus can lead to chronic infections by forming the biofilm on the surface of medical implants [41]. Herein, a majority of the S. aureus isolates were strong biofilm formers, especially MRSA, which should be brought to the attention of Chinese pediatricians. In addition, agr dysfunction has been linked to increased biofilm formation and enhanced colonization ability previously [11]; however, this phenomenon was inconsistent with that in the current study and could be attributed to the small sample size of agr dysfunctional isolates.

Both PVL and biofilm are major virulence factors of $S$. aureus. Previous studies found that some secreted virulence factors are closely related to biofilm formation, such as phenol-soluble modulin $\alpha$ (PSM $\alpha)$ [42]. Thus, we tried to explore the correlation between PVL and 
Table 4 Biofilm formation ability of MRSA and MSSA regarding agr functionality [N (\%)]

\begin{tabular}{lllll}
\hline & No. of isolates & WBF & MBF & SBF \\
\hline MRSA & 211 & $1(0.47)$ & $23(10.90)$ & $187(88.63)$ \\
Functional agr & 203 & $1(0.49)$ & $23(11.33)$ & $179(88.18)$ \\
Dysfunctional agr & 8 & 0 & 0 & $8(100.00)$ \\
MSSA & 58 & $2(3.45)$ & $23(39.66)$ & $33(56.90)$ \\
Functional agr & 56 & $2(3.57)$ & $22(39.29)$ & $32(57.14)$ \\
Dysfunctional agr & 2 & 0 & $1(50.00)$ & $1(50.00)$ \\
Total & 269 & $3(1.11)$ & $48(17.84)$ & $218(81.04)$ \\
\hline
\end{tabular}

WBF weak biofilm formation, $M B F$ moderate biofilm formation, $S B F$ strong biofilm formation biofilm formation. However, no such association was found in both MRSA and MSSA isolates. A previous meta-analysis demonstrated that PVL strains are rare in colonizing isolates as compared to isolates causing skin and soft-tissue infections [43]. These results indicated that PVL was primarily associated with disease rather than biofilm formation and colonization.

Intriguingly, we found that agr dysfunctional isolates were more resistant than isolates with functional $a g r$. However, at present, agr dysfunction is the result or cause of drug resistance remains unclear, while methicillin resistance might lead to $a g r$ dysfunction. Rudkin et al. [44] found that the expression of mecA (major
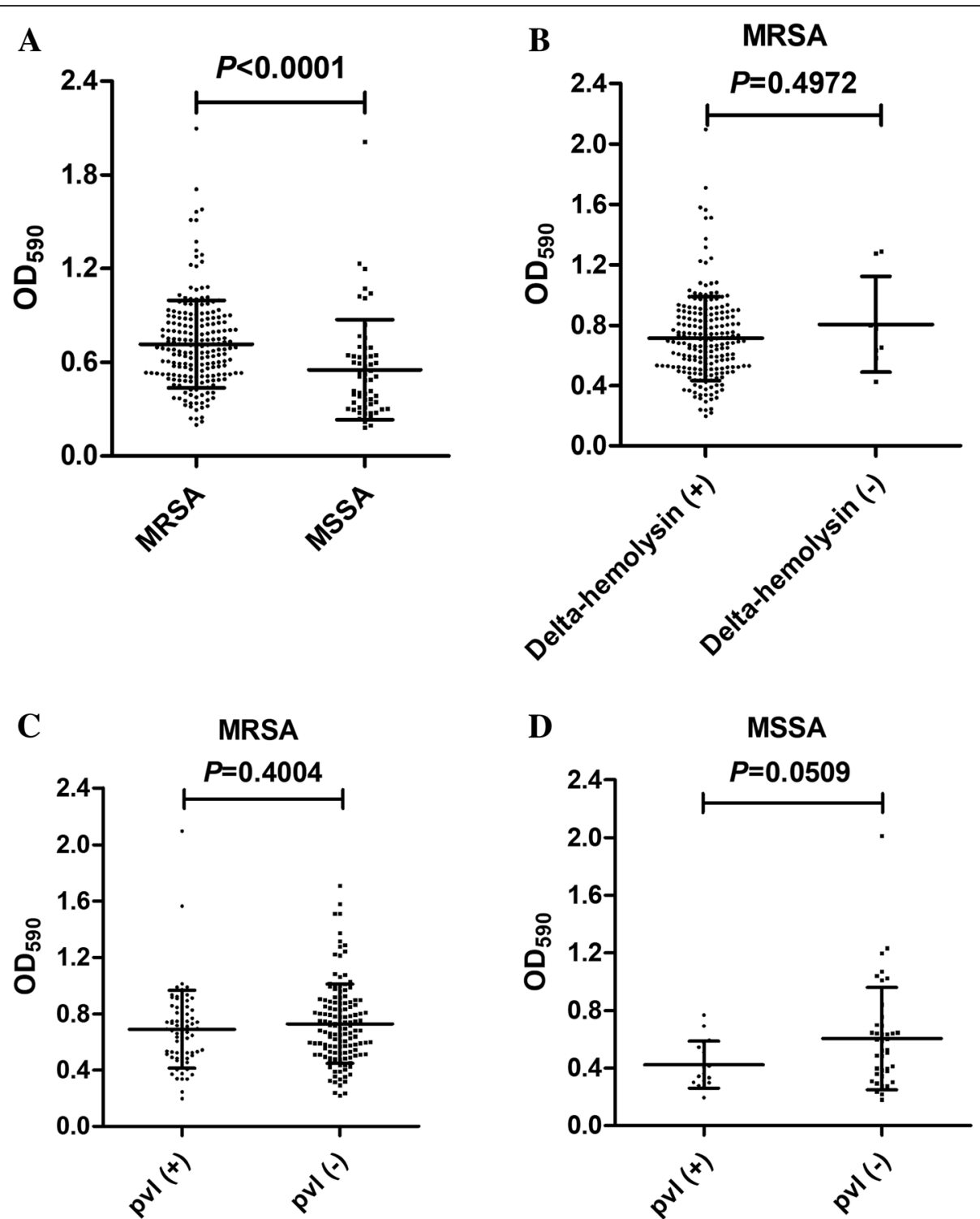

Fig. 2 Biofilm formation ability of S. aureus. a Comparison between MRSA and MSSA isolates. b Comparison between agr dysfunctional and agr functional isolates among MRSA. c Comparison between pvl (+) and pvl (-) isolates among MRSA. d Comparison between pvl (+) and pvl (-) isolates among MSSA. OD 590 , optical density at $590 \mathrm{~nm}$ 
Table 5 Non-susceptibility rates of S. aureus in pediatric population in China [N (\%)]

\begin{tabular}{|c|c|c|c|c|c|c|c|c|c|c|c|c|}
\hline & $\begin{array}{l}\text { No. of } \\
\text { isolates }\end{array}$ & PEN & OXA & ERY & CLI & TET & GEN & $\mathrm{CHL}$ & RIF & CIP & SXT & MDR \\
\hline MRSA & 211 & $\begin{array}{l}207 \\
(98.10)\end{array}$ & $\begin{array}{l}176 \\
(83.41)\end{array}$ & $\begin{array}{l}208 \\
(98.58)\end{array}$ & $\begin{array}{l}181 \\
(85.78)\end{array}$ & $\begin{array}{l}103 \\
(48.82)\end{array}$ & $\begin{array}{l}38 \\
(18.01)\end{array}$ & $\begin{array}{l}121 \\
(57.35)\end{array}$ & $\begin{array}{l}22 \\
(10.43)\end{array}$ & $\begin{array}{l}65 \\
(30.81)\end{array}$ & $5(2.37)$ & $\begin{array}{l}169 \\
(80.09)\end{array}$ \\
\hline $\begin{array}{l}\text { Dysfunctional } \\
\text { agr }\end{array}$ & 8 & $8(100.0)$ & $7(87.50)$ & $8(100.0)$ & $7(87.50)$ & $6(75.00)$ & $\begin{array}{l}6 \\
(75.00)\end{array}$ & $3(37.50)$ & $\begin{array}{l}1 \\
(12.50)\end{array}$ & $\begin{array}{l}7 \\
(87.50)\end{array}$ & $\begin{array}{l}3 \\
(37.50)\end{array}$ & $7(87.50)$ \\
\hline Functional agr & 203 & $\begin{array}{l}199 \\
(98.03)\end{array}$ & $\begin{array}{l}169 \\
(83.25)\end{array}$ & $\begin{array}{l}200 \\
(98.52)\end{array}$ & $\begin{array}{l}174 \\
(85.71)\end{array}$ & $\begin{array}{l}97 \\
(47.78)\end{array}$ & $\begin{array}{l}32 \\
(15.76)\end{array}$ & $\begin{array}{l}118 \\
(58.13)\end{array}$ & $\begin{array}{l}21 \\
(10.34)\end{array}$ & $\begin{array}{l}58 \\
(28.57)\end{array}$ & $2(0.99)$ & $\begin{array}{l}162 \\
(79.80)\end{array}$ \\
\hline${ }^{a} P$-value & & 1.0000 & 1.0000 & 1.0000 & 1.0000 & 0.1627 & 0.0005 & 0.2904 & 0.5921 & 0.0013 & 0.0003 & 1.0000 \\
\hline MSSA & 58 & $\begin{array}{l}54 \\
(93.10)\end{array}$ & 0 & $\begin{array}{l}58 \\
(100.00\end{array}$ & $\begin{array}{l}19 \\
(32.76)\end{array}$ & $\begin{array}{l}11 \\
(18.97)\end{array}$ & $\begin{array}{l}12 \\
(20.69)\end{array}$ & $\begin{array}{l}33 \\
(56.90)\end{array}$ & 0 & $\begin{array}{l}11 \\
(18.97)\end{array}$ & 0 & $\begin{array}{l}43 \\
(74.14)\end{array}$ \\
\hline Total & 269 & $\begin{array}{l}261 \\
(97.03)\end{array}$ & $\begin{array}{l}176 \\
(65.43)\end{array}$ & $\begin{array}{l}266 \\
(98.89)\end{array}$ & $\begin{array}{l}200 \\
(74.35)\end{array}$ & $\begin{array}{l}114 \\
(42.38)\end{array}$ & $\begin{array}{l}50 \\
(18.59)\end{array}$ & $\begin{array}{l}154 \\
(57.25)\end{array}$ & $\begin{array}{l}22 \\
(8.18)\end{array}$ & $\begin{array}{l}76 \\
(28.25)\end{array}$ & $5(1.86)$ & $\begin{array}{l}212 \\
(78.81)\end{array}$ \\
\hline${ }^{b} P$-value & & 0.0688 & $<0.0001$ & 1.0000 & $<0.0001$ & $<0.0001$ & 0.7033 & 1.0000 & 0.0057 & 0.0990 & 0.5883 & 0.3648 \\
\hline
\end{tabular}

All isolates were susceptible to vancomycin and linezolid, and hence, not listed in the table

PEN Penicillin, OXA oxacillin, ERY Erythromycin, CLI Clindamycin, TET Tetracycline, GEN Gentamicin, CHL Chloramphenicol, CIP Ciprofloxacin, RIF Rifampin, SXT Trimethoprim-sulfamethoxazole

$M D R$ multi-drug resistance, MDR-MRSA resistant to $\geq 3$ classes of non- $\beta$-lactam antimicrobials, MDR-MSSA resistant to $\geq 3$ classes of antibiotics including $\beta$-lactam antibiotics

${ }^{a}$ Comparison between agr dysfunctional and agr functional isolates among MRSA

${ }^{b}$ Comparison between MRSA and MSSA isolates

resistance determinant of MRSA) could subtly affect the peptidoglycan structure or its interaction with other cell wall-associated proteins and prevented the detection of autoinducing peptide (AIP), thereby resulting in an unresponsive agr system and the subsequent low-level toxicity. When the type II SCCmec element or only mecA was deleted from an isolate with dysfunctional $a g r$, the agr activity was restored. However, Tsuji et al. [45] suggested that agr dysfunctional might directly influence the acquisition of intermediate resistance to vancomycin after subtherapeutic exposure.

Nonetheless, all S. aureus strains, including the $10 \mathrm{agr}$ dysfunctional isolates, were sensitive to vancomycin. Kim et al. [46] showed that $2 / 12$ initial $a g r$-functional isolates acquired $a g r$ dysfunction during vancomycin therapy for persistent bacteremia, but were still sensitive to vancomycin. In addition, all the 4 strains developed from vancomycin-susceptible $S$. aureus (VSSA) to heterogeneous VISA (hVISA) were initially $a g r$ dysfunctional strains. Therefore, we speculated that agr dysfunctional isolates can more easily adapt to glycopeptide selection pressure than agr functional isolates. Taken together, further studies assessing the correlation between agr dysfunction and antibiotic resistance, especially vancomycin resistance, are imperative.

The agr quorum sensing system has become a new target for developing new antibiotics. Hitherto, many natural and synthetic compounds have been found to interfere with the functions of agr [47]. However, the prevalence of agr dysfunction among $S$. aureus, and the potential correlation between $a g r$ dysfunction and antibiotic resistance indicated that isolates could withstand

Table 6 agr mutations for non-hemolytic isolates

\begin{tabular}{|c|c|c|c|}
\hline Strain & MLST & agr mutations & Predicted results \\
\hline$\overline{R 85^{a}}$ & 59 & $\operatorname{agrc} 62-63$ ins $t$ & Frameshift-truncated AgrC \\
\hline $\mathrm{R} 90^{\mathrm{a}}$ & 59 & agrA g443a & Asp> Gly at aa 148 in AgrA \\
\hline $11-3^{b}$ & 239 & agrD a48g; agrC t884c & No aa change in agrD; lle > Thr at aa 295 in AgrC \\
\hline $11-6^{b}$ & 239 & None & None \\
\hline $11-15^{c}$ & 9 & agrB g519a; Many mutations in agrC and agrA & No aa change in AgrB; Many aa changes in AgrC and AgrA \\
\hline $12-31^{b}$ & 239 & None & None \\
\hline $12-98^{b}$ & 1296 & agrD a7g; agrC a704c + a875g & Thr > Ala at aa 3 in AgrD; Tyr > Ser at aa $235+$ Asn $>$ Ser at aa 292 in AgrC \\
\hline SA2017112b & 239 & $\operatorname{agrA~t615c}$ & No aa change in AgrA \\
\hline $\mathrm{S} 14^{d}$ & 398 & $\operatorname{agrC} c 415 t ; \operatorname{agrA} c 263 t$ & Pro $>$ Ser at aa 139 in AgrC; Thr > Met at aa 88 inAgrA \\
\hline $578^{e}$ & 22 & $\operatorname{agrc} 434-435$ ins $t$ & Frameshift-truncated AgrC \\
\hline
\end{tabular}

${ }^{a}$ Compared to SA957 genome (NC_022442.1); ${ }^{b}$ Compared to NCTC8325 genome (CP000253.1); ${ }^{c}$ Compared to N315 genome (NC_002745.2); ${ }^{d}$ Compared to ST398 genome (NC_017333.1); ${ }^{e}$ Compared to H-EMRSA-15 genome (CP007659.1). ins, insertion; del, deletion; aa, amino acid; -, not applicable 
drug interference with agr functionality emerge rapidly. Strikingly, the promotion effects of agr dysfunction on biofilm formation may pose a great threat for patients using indwelling devices. Thus, additional studies are needed to explore the feasibility of $a g r$ system as a new target for antimicrobial agents.

Herein, we found that the $a g r$ sequence was not only associated with the agr group but also to the MLST types. Thus, the agr group and MLST types were considered while detecting the occurrence of mutation in the current agr dysfunctional isolates. Furthermore, agr dysfunctional isolates detected mutations that mainly occurred in $\operatorname{agr} C$ and $\operatorname{agr} A$, leading to the inactivation of the auto-activation circuit and RNAIII expression [48]. However, no mutation or synonymous mutation or no mutations were also detected in our study, which suggested the involvement of additional mechanisms in $a g r$ dysfunction. Interestingly, mecA expression $[49,50]$ and abnormal expression of regulators of agr (sarA) can also be the cause of agr dysfunction [11].

Nevertheless, the current study had limitations. First, the sample size was relatively small. Second, this was a single-center study, and all the isolates were collected from one hospital. However, the hospital serves the whole of China, and $>60 \%$ of the hospitalized children come from all over the country. Therefore, the present study is still representative in China. Third, some MRSA strains used in this study have been sub-cultured 2 or 3 times since first collected, and multiple subcultures and long-term cryopreservation might have affected the characteristics of the strain.

\section{Conclusions}

In summary, agr dysfunction was not common in pediatric populations in China, and ST59-SCCmec IV was associated with a low prevalence of agr dysfunction as compared to the ST239-SCCmec III isolates. Agr dysfunctional isolates were always healthcare-associated and multidrug resistant. Except for the gene mutations, other mechanisms might also be involved in agr dysfunction. The agr dysfunction might be a major adaption mechanism of $S$. aureus to antibiotic selection pressure. Thus, an in-depth understanding of $a g r$ dysfunction is an urgent requirement for the development of new antibiotics that target agr expression.

\footnotetext{
Abbreviations

agr: accessory gene regulator; Al: Intra-abdominal infection; BJl: Bone and joint infection; BSI: Bloodstream infection; CA: Community-associated; CHL: Chloramphenicol; CIP: Ciprofloxacin; CLI: Clindamycin; CLSI: Clinical and Laboratory Standards Institute; CNSI: Central nervous system infection; ERY: Erythromycin; GEN: Gentamicin; HA: Healthcare-associated; hVISA: heterogeneous VISA; IE: Infective endocarditis; LA-MRSA: Livestockassociated MRSA; LNZ: Linezolid; MDR: Multidrug resistance; MLST: Multilocus sequence typing; MRSA: Methicillin-resistant S. aureus; MSCRAMMs: Microbial surface components recognizing adhesive matrix molecules; OD: Optical density; ODc: Cut-off OD value; OXA: Oxacillin; PCR: Polymerase chain
}

reaction; PEN: Penicillin G; pvl: panton-Valentine leukocidin; RIF: Rifampin; S. aureus: Staphylococcus aureus; SCCmec: Staphylococcal cassette chromosome mec; SP: Severe pneumonia; spa: staphylococcal protein A; SSTI: Skin and soft tissue infection; SXT: Sulphamethoxazole/trimethoprim; TCP: Tissue culture plate method; TET: Tetracycline; TSB: Tryptic soy broth; VAN: Vancomycin; VISA: Vancomycin intermediate S. aureus; VSSA: Vancomycin-susceptible S. aureus

\section{Acknowledgments}

We thank the research group of Xuzhuang Shen, who worked in Beijing Pediatric Research Institute in China, for supplying the S. aureus standard strains used in this study.

\section{Funding}

This study was funded by the Beijing Natural Science Foundation (No.7172075) and the National Natural Science Foundation of China (No. 81571948).

\section{Availability of data and materials}

All data generated or analysed during this study are included in this published article.

\section{Authors' contributions}

XY detected the biofilm formation ability, performed MLST, SCCmec and spa typing, analyzed the data, and drafted the manuscript. FD analyzed the data and drafted the article. SQ designed the study and revised the article. KY statistically analyzed the data and revised the article. LW and YL performed MLST and spa typing. WS analyzed the data and drafted the article. JZ, WZ, $\mathrm{HX}$, and $\mathrm{HZ}$ collected and identified the clinical strains of S. aureus and analyzed the data. All authors had read and approved the final manuscript.

\section{Ethics approval and consent to participate}

This study mainly used the bacterial isolates from the biological specimens obtained during the clinical diagnosis and management of patients without threat to the subjects' rights and health. The applications for the exemption of written informed content and ethical review were approved by the Ethics Committee of Beijing Children's Hospital Affiliated to Capital Medical University according to national regulations (No. 2016-93, 23/06/2016). Thus, only verbal consent was obtained from the patient's legal guardian. This study also obtained clearance from the Institutional Biosafety Committee (IBSC) ([2017] No.43)

Consent for publication

Not applicable.

\section{Competing interests}

The authors declare that they have no competing interests.

\section{Publisher's Note}

Springer Nature remains neutral with regard to jurisdictional claims in published maps and institutional affiliations.

\section{Author details}

${ }^{1}$ Pediatric Intensive Care Unit, Beijing Children's Hospital, Capital Medical University, National Center for Children's Health, No.56 Nan-Li-Shi Road Beijing 100045, China. 'Bacteriology Laboratory, Beijing Children's Hospital, Capital Medical University, National Center for Children's Health, Beijing 100045, China. ${ }^{3}$ MOE Key Laboratory of Major Diseases in Children, National Key Discipline of Pediatrics (Capital Medical University), National Clinical Research Center for Respiratory Diseases, Beijing Key Laboratory of Pediatric Respiratory Infection Diseases, Beijing Pediatric Research Institute, Beijing Children's Hospital, Capital Medical University, National Center for Children's Health, Beijing 100045, China. ${ }^{4}$ Present address: No. 56 Nan-li-shi Road, Beijing 100045, China.

Received: 13 August 2018 Accepted: 26 April 2019

Published online: 14 May 2019

\section{References}

1. Shrestha B. Review on Panton valentine leukocidin toxin carriage among Staphylococcus aureus. J Nepal Health Res Counc. 2013;11:305-12. 
2. Oyama T, Miyazaki M, Yoshimura M, Takata T, Ohjimi H, Jimi S. Biofilmforming methicillin-resistant Staphylococcus aureus survive in Kupffer cells and exhibit high virulence in mice. Toxins. 2016;8(7):E198.

3. Chung PY, Toh YS. Anti-biofilm agents: recent breakthrough against multidrug resistant Staphylococcus aureus. Pathog Dis. 2014;70:231-9.

4. Yarwood JM, Schlievert PM. Quorum sensing in Staphylococcus infections. J Clin Invest. 2003;112:1620-5.

5. Cheung AL, Eberhardt KJ, Chung E, Yeaman MR, Sullam PM, Ramos M, et al. Diminished virulence of a sar- / agr- mutant of Staphylococcus aureus in the rabbit model of endocarditis. J Clin Invest. 1994;94:1815-22.

6. Cheung GY, Wang R, Khan BA, Sturdevant DE, Otto M. Role of the accessory gene regulator agr in community-associated methicillin-resistant Staphylococcus aureus pathogenesis. Infect Immun. 2011;79:1927-35.

7. Kobayashi SD, Malachowa N, Whitney AR, Braughton KR, Gardner DJ, Long $D$, et al. Comparative analysis of USA300 virulence determinants in a rabbit model of skin and soft tissue infection. J Infect Dis. 2011;204:937-41.

8. Montgomery CP, Boyle-Vavra S, Daum RS. Importance of the global regulators Agr and SaeRS in the pathogenesis of CA-MRSA USA300 infection. PLoS One. 2010:5:e15177.

9. Gillaspy AF, Hickmon SG, Skinner RA, Thomas JR, Nelson CL, Smeltzer MS. Role of the accessory gene regulator (agr) in pathogenesis of staphylococcal osteomyelitis. Infect Immun. 1995;63:3373-80.

10. Abdelnour A1, Arvidson S, Bremell T, Rydén C, Tarkowski A. The accessory gene regulator (agr) controls Staphylococcus aureus virulence in a murinearthritis model. Infect Immun. 1993;61:3879-85.

11. Eerreira FA, Souza RR, de Sousa Moraes B, de Amorim Ferreira AM, Américo MA, Fracalanzza SE, et al. Impact of agr dysfunction on virulence profiles and infections associated with a novel methicillin-resistant Staphylococcus aureus (MRSA) variant of the lineage ST1-SCCmec IV. BMC Microbiol. 2013; 13:93.

12. Chong YP, Park SJ, Kim HS, Kim ES, Kim MN, Park KH, et al. Persistent Staphylococcus aureus bacteremia:a prospective analysis of risk factors, outcomes, and microbiologic and genotypic characteristics of isolates. Medicine (Baltimore). 2013:92:98-108.

13. Butterfield JM, Tsuji BT, Brown J, Ashley ED, Hardy D, Brown K, et al. Predictors of agr dysfunction in methicillin-resistant Staphylococcus aureus (MRSA) isolates among patients with MRSA bloodstream infections. Antimicrob Agents Chemother. 2011;55:5533-7.

14. Park SY, Chong YP, Park HJ, Park KH, Moon SM, Jeong JY, et al. agr dysfunction and persistent methicillin- resistant Staphylococcus aureus bacteremia inpatients with removed eradicable foci. Infection. 2013;41:111-9.

15. Sakoulas G, Moise PA, Rybak MJ. Accessory gene regulator dysfunction: an advantage for Staphylococcus aureus in health-care settings? J Infect Dis. 2009;199:1558-9.

16. Cameron DR, Howden BP, Peleg AY. The interface between antibiotic resistance and virulence in Staphylococcus aureus and its impact upon clinical outcomes. Clin Infect Dis. 2011;53:576-82.

17. Valour F, Rasigade JP, Trouillet-Assant S, Gagnaire J, Bouaziz A, Karsenty J, et al. Delta-toxin production deficiency in Staphylococcus aureus: a diagnostic marker of bone andjoint infection chronicity linked with osteoblast invasion and biofilm formation. Clin Microbiol Infect. 2015;21:568.e1-11.

18. Schweizer ML, Furuno JP, Sakoulas G, Johnson JK, Harris AD, Shardell MD, et al. Increased mortality with accessory gene regulator (agr) dysfunction in Staphylococcus aureus among bacteremic patients. Antimicrob Agents Chemother. 2011;55:1082-7.

19. Fowler VG Jr, Sakoulas G, McIntyre LM, Meka VG, Arbeit RD, Cabell CH, et al. Persistent bacteremia due to methicillin-resistant Staphylococcus aureus infection is associated with agr dysfunction and low-levels in vitro resistance to thrombin-induced platelet microbicidal protein. J Infect Dis. 2004:190:1140-9.

20. Kateete DP, Kimani CN, Katabazi FA, Okeng A, Okee MS, Nanteza A, et al. Identification of Staphylococcus aureus: DNase and mannitol salt agar improve the efficiency of the tube coagulase test. Ann Clin Microbiol Antimicrob. 2010:9:23.

21. Petersson AC, Olsson-Liljequist B, Miörner $H$, Haeggman S. Evaluating the usefulness of spa typing, in comparison with pulsed-field gel electrophoresis, for epidemiological typing of methicillin-resistant Staphylococcus aureus in a low-prevalence region in Sweden 2000-2004. Clin Microbiol Infect. 2010;16:456-62.

22. Li S, Sun J, Zhang J, Li X, Tao X, Wang L, et al. Comparative analysis of the virulence characteristics of epidemic methicillin-resistant Staphylococcus aureus (MRSA) strains isolated from Chinese children: ST59 MRSA highly expresses core gene-encoded toxin. APMIS. 2014;122:101-14.

23. Bal AM, Coombs GW, Holden MTG, Lindsay JA, Nimmo GR, Tattevin P, et al. Genomic insights into the emergence and spread of international clones of healthcare-, community- and livestock- associated meticillin-resistant Staphylococcus aureus: blurring of the traditional definitions. J Glob Antimicrob Resist. 2016;6:95-101.

24. Bronner S, Monteil H, Prévost G. Regulation of virulence determinants in Staphylococcus aureus: complexity and applications. FEMS Microbiol Rev. 2004;28:183-200.

25. Traber KE, Lee E, Benson S, Corrigan R, Cantera M, Shopsin B, et al. agr function in clinical Staphylococcus aureus isolates. Microbiology. 2008;154:2265-74.

26. Robinson DA, Monk AB, Cooper JE, Feil EJ, Enright MC. Evolutionary genetics of the accessory gene regulator (agr) locuc in Sthaphylococcus aureus. J Bacteriol. 2005;187:8312-21.

27. Enright MC, Day NP, Davies CE, Peacock SJ, Spratt BG. Multilocus sequence typing for characterization of methicillin-resistant and methicillin-susceptible clones of Staphylococcus aureus. J Clin Microbiol. 2000;38:1008-15.

28. Koreen L, Ramaswamy SV, Graviss EA, Naidich S, Musser JM, Kreiswirth BN. spa typing method for discriminating among Staphylococcus aureus isolates: implications for use of a single marker to detect genetic micro- and macrovariation. J Clin Microbiol. 2004;42:792-9.

29. Gilot P, Lina G, Cochard T, Poutrel B. Analysis of the genetic variability of genes encoding the RNAlll-activating components Agr and TRAP in a population of Staphylococcus aureus strains isolated from cows with mastitis. J Clin Microbiol. 2002:40:4060-7.

30. Milheiriço C, Oliveira DC, de Lencastre H. Multiplex PCR strategy for subtyping the staphylococcal cassette chromosome mec type IV inmethicillin-resistant Staphylococcus aureus: 'SCCmec IV multiplex. J Antimicrob Chemother. 2007;60:42-8.

31. Jarraud S, Mougel C, Thioulouse J, Lina G, Meugnier H, Forey F, et al. Relationships between Staphylococcus aureus genetic background, virulence factors, agr groups (alleles), and human disease. Infect Immun. 2002;70:631-41.

32. Yang X, Qian S, Yao K, Wang L, Liu Y, Dong F, et al. Multiresistant ST59-SCCmec IV-t437 clone with strong biofilm-forming capacity was identified predominantly in MRSA isolated from Chinese children. BMC Infect Dis. 2017;17:733.

33. Wiegand I, Hilpert K, Hancock RE. Agar and broth dilution methods to determine the minimal inhibitory concentration (MIC) of antimicrobial substancs. Nat Protoc. 2008;3:163-75.

34. CLSI, editor. Performance Standards for Antimicrobial Susceptibility Testing. In: CLSI supplement M100S. 26th ed. Wayne: Clinical and Laboratory Standards Institute. p. 2016.

35. Shopsin B, Drlica-Wagner A, Mathema B, Adhikari RP, Kreiswirth BN, Novick RP. Prevalence of agr dysfunction among colonizing Staphylococcus aureus strains. J Infect Dis. 2008;198:1171-4.

36. Le KY, Otto M. Quorum-sensing regulation in staphylococci-an overview. Front Microbiol. 2015;6:1174.

37. Qiao Y, Dong F, Song W, Wang L, Yang Y, Shen X. Hospital- and community-associated methicillin-resistant Staphylococcus aureus: a 6yearsurveillance study of invasive infections in Chinese children. Acta Paediatr. 2013;102:1081-6.

38. Jang HC, Kang SJ, Choi SM, Park KH, Shin JH, Choy HE, et al. Difference in agr dysfunction and reduced vancomycin susceptibility between MRSA bacteremiainvolving SCCmec types IV/IVa and I-III. PLoS One. 2012;7:e49136.

39. Japoni-Nejad A, Rezazadeh M, Kazemian H, Fardmousavi N, van Belkum A, Ghaznavi-Rad E. Molecular characterization of the first community-acquired methicillin-resistant Staphylococcus aureus strains from Central Iran. Int J Infect Dis. 2013;17:e949-54.

40. Monecke S, Coombs G, Shore AC, Coleman DC, Akpaka P, Borg M, et al. A field guide to pandemic, epidemic and sporadic clones of methicillinresistant Staphylococcus aureus. PLoS One. 2011;6:e17936.

41. Vergara-lrigaray M, Valle J, Merino N, Latasa C, Garcia B, Ruiz de Los Mozos I, et al. Relevant role of fibronectin-binding proteins in Staphylococcus aureus biofilm-associated foreign-body infections. Infect Immun. 2009;77:3978-91.

42. Le KY, Dastgheyb S, Ho TV, Otto M. Molecular determinants of staphylococcal biofilm dispersal and structuring. Front Cell Infect Microbiol. 2014;4:167.

43. Shallcross LJ, Fragaszy E, Johnson AM, Hayward AC. The role of the Pantonvalentine leucocidin toxin in staphylococcal disease: a systematic reviewand meta-analysis. Lancet Infect Dis. 2013;13:43-54.

44. Rudkin JK, Edwards AM, Bowden MG, Brown EL, Pozzi C, Waters EM, et al. Methicillin resistance reduces the virulence of healthcare-associated 
methicillin-resistantStaphylococcus aureus by interfering with the agr quorum sensing system. J Infect Dis. 2012;205:798-806.

45. Tsuji BT, Rybak MJ, Lau KL, Sakoulas G. Evaluation of accessory gene regulator (agr) group and function in the proclivity towardsvancomycin intermediate resistance in Staphylococcus aureus. Antimicrob Agents Chemother. 2007;51:1089-91

46. Kim T, Kim ES, Park SY, Sung H, Kim MN, Kim SH, et al. Phenotypic changes of methicillin-resistant Staphylococcus aureus during vancomycin therapy for persistent bacteraemia and related clinical outcome. Eur J Clin Microbiol Infect Dis. 2017;36:1473-81.

47. Khan BA, Yeh AJ, Cheung GY, Otto M. Investigational therapies targeting quorum-sensing for the treatment of Staphylococcus aureus infections. Expert Opin Investig Drugs. 2015;24:689-704

48. Painter KL, Krishna A, Wigneshweraraj S, Edwards AM. What role does the quorum-sensing accessory gene regulator system play during Staphylococcus aureus bacteremia? Trends Microbiol. 2014;22:676-85.

49. Pozzi C, Waters EM, Rudkin JK, Schaeffer CR, Lohan AJ, Tong P, et al. Methicillin resistance alters the biofilm phenotype and attenuates virulence in Staphylococcus aureus device-associated infections. PLoS Pathog. 2012;8: e1002626.

50. Rudkin JK, Edwards AM, Bowden MG, Brown EL, Pozzi C, Waters EM, et al. Methicillin resistance reduces the virulence of healthcare-associated methicillin-resistant Staphylococcus aureus by interfering with the agr quorum sensing system. J Infect Dis. 2012;205:798-806.

Ready to submit your research? Choose BMC and benefit from:

- fast, convenient online submission

- thorough peer review by experienced researchers in your field

- rapid publication on acceptance

- support for research data, including large and complex data types

- gold Open Access which fosters wider collaboration and increased citations

- maximum visibility for your research: over $100 \mathrm{M}$ website views per year

At $\mathrm{BMC}$, research is always in progress.

Learn more biomedcentral.com/submissions 